\title{
On $\frac{1}{w}+\frac{1}{x}+\frac{1}{y}+\frac{1}{z}=\frac{1}{2}$ and some of its generalizations
}

Tingting Bai $1^{*}$

\section{${ }^{*}$ Correspondence:} baiting-ting@hotmail.com ${ }^{1}$ College of Mathematics and Information Science, Baoji University of Arts and Sciences, Baoji, China

\begin{abstract}
In this paper, we give a straightforward approach to obtaining the solution of the Diophantine equation $\frac{1}{w}+\frac{1}{x}+\frac{1}{y}+\frac{1}{z}=\frac{1}{2}$. We also establish that the Diophantine equation $\frac{1}{w}+\frac{1}{x}+\frac{1}{y}+\frac{1}{z}=\frac{m}{n}$ for any two positive integers $m$ and $n$ has only a finite number of solutions in the positive integers $w, x, y$, and $z$.
\end{abstract}

MSC: 11068

Keywords: Diophantine equation; Integer solution

\section{Introduction and preliminaries}

The unit fractional decomposition of certain rational fractions was considered one of fascinating problems by the ancient Egyptians. One of such problems is a well-known conjecture due to Erdos and Strauss in 1948. They conjectured that for each $n>1$, the Diophantine equation

$$
\frac{4}{n}=\frac{1}{x}+\frac{1}{y}+\frac{1}{z}
$$

has a solution in positive integers $x, y$, and $z$. Although it has been investigated by many mathematicians, the conjecture is still open. A good number of partial results have been obtained by several mathematicians (see [1, 3, 5, 6, 8, 9]). Mordell [7] has proven that the conjecture is true for all $n$ except possibly cases in which $n$ is congruent to $1,121,169,289,361,529(\bmod 840)$. For the extensive literature + Sierpinski, Schinzel, and others, we refer the reader to [4]. Recently, Elsholtz and Tao [2] investigated the average behavior of a number of positive integer solutions in $x, y$, and $z$ of the above Diophantine equation in the case when $n$ is prime.

In this paper, we consider an analogue of the above conjecture of Erdos and Strauss. More precisely, we study the Diophantine equation

$$
\frac{1}{w}+\frac{1}{x}+\frac{1}{y}+\frac{1}{z}=\frac{1}{2}
$$

and give a detailed solution to Eq. (1.1). We also draw our attention to some of the generalizations of Eq. (1.1). We use elementary arguments and inequalities to prove the results.

(c) The Author(s) 2018. This article is distributed under the terms of the Creative Commons Attribution 4.0 International License (http://creativecommons.org/licenses/by/4.0/), which permits unrestricted use, distribution, and reproduction in any medium, provided you give appropriate credit to the original author(s) and the source, provide a link to the Creative Commons license, and indicate if changes were made. 


\section{Main results and discussion}

In this section, we first find the solutions in positive integers $x, y, z$, and $w$ of Eq. (1.1).

Without loss of generality, we may assume that $w \leq x \leq y \leq z$. Then Eq. (1.1) gives:

(a) $\frac{1}{w}<\frac{1}{2}$ and thus $w \geq 3$;

(b) $\frac{1}{w}+\frac{1}{x}+\frac{1}{y}+\frac{1}{z} \geq \frac{4}{z}$ and thus $z \geq 8$; and

(c) $\frac{1}{w}+\frac{1}{x}+\frac{1}{y}+\frac{1}{z} \leq \frac{4}{w}$ and thus $w \leq 8$.

Using (a) and (c), we see that $w \in\{3,4,5,6,7,8\}$. Thus Eq. (1.1) can be rewritten as follows:

When $w=3$,

$$
\frac{1}{x}+\frac{1}{y}+\frac{1}{z}=\frac{1}{6}
$$

When $w=4$,

$$
\frac{1}{x}+\frac{1}{y}+\frac{1}{z}=\frac{1}{4},
$$

When $w=5$,

$$
\frac{1}{x}+\frac{1}{y}+\frac{1}{z}=\frac{3}{10}
$$

When $w=6$,

$$
\frac{1}{x}+\frac{1}{y}+\frac{1}{z}=\frac{1}{3},
$$

When $w=7$,

$$
\frac{1}{x}+\frac{1}{y}+\frac{1}{z}=\frac{5}{14},
$$

When $w=8$,

$$
\frac{1}{x}+\frac{1}{y}+\frac{1}{z}=\frac{3}{8}
$$

We now find the solution of Eq. (2.1).

Clearly $\frac{1}{x}<\frac{1}{6}$ and thus $x>6$.

Under the assumption $x \leq y \leq z$, Eq. (2.1) gives $\frac{1}{x}+\frac{1}{y}+\frac{1}{z} \leq \frac{3}{x}$ and thus $x \leq 18$.

Hence $x \in\{7,8,9,10,11,12,13,14,15,16,17,18\}$, and thus we have the following cases: For $x=7$,

$$
\frac{1}{y}+\frac{1}{z}=\frac{1}{42},
$$

For $x=8$,

$$
\frac{1}{y}+\frac{1}{z}=\frac{1}{24},
$$


For $x=9$,

$$
\frac{1}{y}+\frac{1}{z}=\frac{1}{18}
$$

For $x=10$,

$$
\frac{1}{y}+\frac{1}{z}=\frac{1}{15}
$$

For $x=11$,

$$
\frac{1}{y}+\frac{1}{z}=\frac{5}{66}
$$

For $x=12$,

$$
\frac{1}{y}+\frac{1}{z}=\frac{1}{12}
$$

For $x=13$,

$$
\frac{1}{y}+\frac{1}{z}=\frac{7}{78}
$$

For $x=14$,

$$
\frac{1}{y}+\frac{1}{z}=\frac{2}{21}
$$

For $x=15$,

$$
\frac{1}{y}+\frac{1}{z}=\frac{1}{10}
$$

For $x=16$,

$$
\frac{1}{y}+\frac{1}{z}=\frac{5}{48},
$$

For $x=17$,

$$
\frac{1}{y}+\frac{1}{z}=\frac{11}{102}
$$

For $x=18$,

$$
\frac{1}{y}+\frac{1}{z}=\frac{1}{9}
$$

Equations (2.7), (2.8), (2.9), (2.10), (2.12), (2.14), (2.15), (2.18) may be written as follows:

$$
(y-42)(z-42)=1764 \text {, }
$$




$$
\begin{aligned}
& (y-24)(z-24)=576, \\
& (y-18)(z-18)=324, \\
& (y-15)(z-15)=225, \\
& (y-12)(z-12)=144, \\
& (y-10)(z-10)=100, \\
& (y-9)(z-9)=81 .
\end{aligned}
$$

Under the assumption $x \leq y \leq z$, Eq. (2.1) gives $\frac{1}{x}+\frac{1}{y}+\frac{1}{z} \geq \frac{3}{z}$ and thus

$$
z \geq 18
$$

Under inequality (2.13) and $(y-42) \leq(z-42)$, Eq. $\left(2.7^{\prime}\right)$ leads to:

$$
\begin{array}{ll}
(y-42)=1, & (z-42)=1764, \\
(y-42)=2, & (z-42)=882, \\
(y-42)=3, & (z-42)=588, \\
(y-42)=4, & (z-42)=441, \\
(y-42)=6, & (z-42)=294, \\
(y-42)=7, & (z-42)=252, \\
(y-42)=9, & (z-42)=196, \\
(y-42)=12, & (z-42)=147, \\
(y-42)=14, & (z-42)=126, \\
(y-42)=18, & (z-42)=98 .
\end{array}
$$

Thus $(y, z) \in\{(43,1806),(44,924),(45,630),(46,483),(48,336),(49,294),(51,238)$, $(54,189),(56,168),(60,140)\}$.

Hence Eq. (2.7') leads to the following solutions of Eq. (1.1):

$$
\begin{aligned}
(w, x, y, z) \in & \{(3,7,43,1806),(3,7,44,924),(3,7,45,630),(3,7,46,483),(3,7,48,336), \\
& (3,7,49,294),(3,7,51,238),(3,7,54,189),(3,7,56,168),(3,7,60,140)\} .
\end{aligned}
$$

Under inequality (2.13) and $(y-24) \leq(z-24)$, Eq. $\left(2.8^{\prime}\right)$ gives the following solutions:

$$
\begin{aligned}
(y, z)= & \{(25,600),(26,312),(27,216),(28,168),(30,120),(32,96),(33,88),(36,72), \\
& (40,60),(42,56)\} .
\end{aligned}
$$

Hence Eq. (2.8') leads to the following solutions of Eq. (1.1):

$$
(w, x, y, z) \in\{(3,8,25,600),(3,8,26,312),(3,8,27,216),(3,8,28,168),(3,8,30,120),
$$




$$
(3,8,32,96),(3,8,33,88),(3,8,36,72),(3,8,40,60),(3,8,42,56)\}
$$

Under inequality (2.13) and $(y-18) \leq(z-18)$, Eq. (2.9') gives the following solutions:

$$
(y, z) \in\{(19,342),(20,180),(21,126),(22,99),(24,72),(27,54),(30,45),(36,36)\} .
$$

Hence Eq. (2.9') leads to the following solutions of Eq. (1.1):

$$
\begin{aligned}
(w, x, y, z) \in & \{(3,9,19,342),(3,9,20,180),(3,9,21,126),(3,9,22,99),(3,9,24,72), \\
& (3,9,27,54),(3,9,30,45),(3,9,36,36)\} .
\end{aligned}
$$

Under inequality (2.13) and $(y-15) \leq(z-15)$, Eq. $\left(2.10^{\prime}\right)$ gives the following solutions:

$$
(y, z) \in\{(16,240),(18,90),(20,60),(24,40),(30,30)\} .
$$

Hence Eq. (2.10') leads to the following solutions of Eq. (1.1):

$$
(w, x, y, z) \in\{(3,10,16,240),(3,10,18,90),(3,10,20,60),(3,10,24,40),(3,10,30,30)\} .
$$

Under inequality (2.13) and $(y-12) \leq(z-12)$, Eq. $\left(2.12^{\prime}\right)$ gives the following solutions:

$$
(y, z) \in\{(13,156),(14,84),(15,60),(16,48),(18,36),(20,30),(21,28),(24,24)\} .
$$

Hence Eq. (2.12') leads to the following solutions of Eq. (1.1):

$$
\begin{aligned}
(w, x, y, z) \in\{(3,12,13,156),(3,12,14,84),(3,12,15,60),(3,12,16,48),(3,12,18,36), \\
\\
(3,12,20,30),(3,12,21,28),(3,12,24,24)\} .
\end{aligned}
$$

Under inequality (2.13) and $(y-10) \leq(z-10)$, Eq. $\left(2.15^{\prime}\right)$ gives the following solutions:

$$
(y, z) \in\{(11,110),(12,60),(14,35),(15,30),(20,20)\} \text {. }
$$

Hence Eq. (2.15') leads to the following solutions of Eq. (1.1):

$$
(w, x, y, z) \in\{(3,15,11,110),(3,15,12,60),(3,15,14,35),(3,15,15,30),(3,15,20,20)\} .
$$

Under inequality (2.13) and $(y-9) \leq(z-9)$, Eq. $\left(2.18^{\prime}\right)$ gives the following solutions:

$$
(y, z) \in\{(10,90),(12,36),(18,18)\} .
$$

Hence Eq. (2.18') leads to the following solutions of Eq. (1.1):

$$
(w, x, y, z) \in\{(3,18,10,90),(3,18,12,36),(3,18,18,18)\} .
$$

Since $y \leq z, \frac{1}{y}+\frac{1}{z} \leq \frac{2}{y}$ and thus Eq. (2.11) gives

$$
\frac{5}{66} \leq \frac{2}{y} \quad \Rightarrow \quad y \leq 26
$$


Again we have $y \geq x=11$ and hence $y \in\{11,12,13, \ldots, 26\}$. Therefore the solutions of Eq. (2.11) are as follows:

$$
(y, z) \in\{(14,231),(15,110),(22,22)\}
$$

Hence Eq. (2.11) leads to the following solutions of Eq. (1.1):

$$
(w, x, y, z) \in\{(3,11,14,231),(3,11,15,110),(3,11,22,22)\} .
$$

Since $y \leq z, \frac{1}{y}+\frac{1}{z} \leq \frac{2}{y}$ and thus Eq. (2.13) gives

$$
\frac{7}{78} \leq \frac{2}{y} \quad \Rightarrow \quad y \leq 22
$$

Again we have $y \geq x=13$ and hence $y \in\{13,14, \ldots, 22\}$. Therefore the solutions of Eq. (2.13) are as follows:

$$
(y, z)=(13,78) .
$$

Hence Eq. (2.13) leads to the following solutions of Eq. (1.1):

$$
(w, x, y, z)=(3,13,13,78)
$$

Since $y \leq z, \frac{1}{y}+\frac{1}{z} \leq \frac{2}{y}$ and thus Eq. (2.14) gives

$$
\frac{2}{21} \leq \frac{2}{y} \quad \Rightarrow \quad y \leq 21
$$

Again we have $y \geq x=14$ and hence $y \in\{14, \ldots, 21\}$. Therefore the solutions of Eq. (2.14) are as follows:

$$
(y, z) \in\{(14,42),(15,35),(21,21)\} .
$$

Hence Eq. (2.14) leads to the following solutions of Eq. (1.1):

$$
(w, x, y, z) \in\{(3,14,14,42),(3,14,15,35),(3,14,21,21)\} .
$$

Since $y \leq z, \frac{1}{y}+\frac{1}{z} \leq \frac{2}{y}$ and thus Eq. (2.16) gives

$$
\frac{5}{48} \leq \frac{2}{y} \quad \Rightarrow \quad y \leq 19
$$

Again we have $y \geq x=16$ and hence $y \in\{16,17,18,19\}$. Therefore the solutions of Eq. (2.16) are as follows:

$$
(y, z)=(16,24) .
$$


Hence Eq. (2.16) leads to the following solutions of Eq. (1.1):

$$
(w, x, y, z)=(3,16,16,24) .
$$

Since $y \leq z, \frac{1}{y}+\frac{1}{z} \leq \frac{2}{y}$ and thus Eq. (2.17) gives

$$
\frac{11}{102} \leq \frac{2}{y} \quad \Rightarrow \quad y \leq 18
$$

Again we have $y \geq x=17$ and hence $y \in\{17,18\}$.

This shows that Eq. (2.17) has no integer solution and hence Eq. (1.1) too has no integer solutions.

We now solve Eq. (2.2), that is, Eq. (1.1) when $w=4$.

It is clear that $\frac{1}{x}<\frac{1}{4} \Rightarrow x>4$.

Under the assumption $x \leq y \leq z$, Eq. (2.2) gives $\frac{1}{x}+\frac{1}{y}+\frac{1}{z} \leq \frac{3}{x}$ and thus $x \leq 12$.

Hence $x \in\{5,6,7,8,910,11,12\}$ and thus we have the following cases:

For $x=5$,

$$
\frac{1}{y}+\frac{1}{z}=\frac{1}{20},
$$

For $x=6$,

$$
\frac{1}{y}+\frac{1}{z}=\frac{1}{12},
$$

For $x=7$,

$$
\frac{1}{y}+\frac{1}{z}=\frac{3}{28},
$$

For $x=8$,

$$
\frac{1}{y}+\frac{1}{z}=\frac{1}{8},
$$

For $x=9$,

$$
\frac{1}{y}+\frac{1}{z}=\frac{5}{36},
$$

For $x=10$,

$$
\frac{1}{y}+\frac{1}{z}=\frac{3}{20},
$$

For $x=11$,

$$
\frac{1}{y}+\frac{1}{z}=\frac{7}{44},
$$


For $x=12$,

$$
\frac{1}{y}+\frac{1}{z}=\frac{1}{6}
$$

Solving Eqs. (2.14)-(2.21) by the above procedure, we get:

$$
\begin{aligned}
(x, y, z) \in & \{(5,21,420),(2,22,220),(5,24,120),(5,25,100),(5,28,70),(5,30,60), \\
& (6,13,156),(6,14,84),(6,15,60),(6,16,48),(6,18,36),(6,20,30),(6,21,28), \\
& (6,24,24),(8,9,72),(8,10,40),(8,12,24),(8,26,20),(12,7,42), \\
& (12,8,24),(12,9,18),(12,10,15),(12,12,12),(7,10,140),(7,12,42), \\
& (7,14,28),(9,9,36),(9,12,18),(10,10,20),(10,12,15)\} .
\end{aligned}
$$

We now solve Eq. (2.3), that is, Eq. (1.1) when $w=5$.

We see that $\frac{1}{x}<\frac{3}{10} \Rightarrow x \geq 3$.

Under the assumption $x \leq y \leq z$, Eq. (2.3) gives $\frac{1}{x}+\frac{1}{y}+\frac{1}{z} \leq \frac{3}{x}$ and thus $x \leq 10$.

Hence $x \in\{3,4,5,6,7,8,9,10\}$ and thus Eq. (2.3) leads to the following equations:

For $x=3$,

$$
\frac{1}{y}+\frac{1}{z}=-\frac{1}{10}
$$

For $x=4$,

$$
\frac{1}{y}+\frac{1}{z}=\frac{1}{20}
$$

For $x=5$,

$$
\frac{1}{y}+\frac{1}{z}=\frac{1}{10}
$$

For $x=6$,

$$
\frac{1}{y}+\frac{1}{z}=\frac{2}{15}
$$

For $x=7$,

$$
\frac{1}{y}+\frac{1}{z}=\frac{11}{70}
$$

For $x=8$,

$$
\frac{1}{y}+\frac{1}{z}=\frac{7}{40}
$$

For $x=9$,

$$
\frac{1}{y}+\frac{1}{z}=\frac{17}{90}
$$


For $x=10$,

$$
\frac{1}{y}+\frac{1}{z}=\frac{1}{5}
$$

We avoid Eq. (2.22) because it leads to negative solutions.

Solving Eqs. (2.23)-(2.29) by the above procedure, we get:

$$
\begin{aligned}
(x, y, z) \in & \{(4,21,420),(4,22,220),(4,24,120),(4,25,100),(4,28,70),(4,30,60), \\
& (4,40,40),(5,11,110),(5,12,60),(5,14,35),(5,15,30),(5,20,20),(10,6,30), \\
& (10,10,10),(6,8,120),(6,9,45),(6,10,30),(6,12,20),(6,15,15),(7,7,70), \\
& (8,8,20)\} .
\end{aligned}
$$

We now solve Eq. (2.4), that is, Eq. (1.1) when $w=6$.

It is clear that $\frac{1}{x}<\frac{1}{3} \Rightarrow x>3$.

Under the assumption $x \leq y \leq z$, Eq. (2.4) gives $\frac{1}{x}+\frac{1}{y}+\frac{1}{z} \leq \frac{3}{x}$ and thus $x \leq 9$.

Hence $x \in\{4,5,6,7,8,9\}$ and thus Eq. (2.4) leads to the following equations:

For $x=4$,

$$
\frac{1}{y}+\frac{1}{z}=\frac{1}{12}
$$

For $x=5$,

$$
\frac{1}{y}+\frac{1}{z}=\frac{2}{15}
$$

For $x=6$,

$$
\frac{1}{y}+\frac{1}{z}=\frac{1}{6}
$$

For $x=7$,

$$
\frac{1}{y}+\frac{1}{z}=\frac{4}{21}
$$

For $x=8$,

$$
\frac{1}{y}+\frac{1}{z}=\frac{5}{24}
$$

Solving Eqs. (2.30)-(2.26) by the above procedure, we get:

$$
\begin{aligned}
(x, y, z) \in & \{(4,13,156),(4,14,84),(4,15,60),(4,16,48),(4,18,36),(4,20,30),(4,21,28), \\
& (6,7,42),(6,8,24),(6,9,18),(6,10,15),(6,12,12),(6,15,10),(5,8,120), \\
& (5,9,45),(5,10,30),(5,12,20),(5,15,15),(7,7,21),(8,8,12),(8,12,8), \\
& (8,24,6)\} .
\end{aligned}
$$


We now solve Eq. (2.5), that is, Eq. (1.1) when $w=7$.

It is clear that $\frac{1}{x}<\frac{5}{14} \Rightarrow x \geq 2$.

Under the assumption $x \leq y \leq z$, Eq. (2.5) gives $\frac{1}{x}+\frac{1}{y}+\frac{1}{z} \leq \frac{3}{x}$ and thus $x \leq 8$.

Hence $x \in\{2,3,4,5,6,7,8\}$ and thus Eq. (2.5) leads to the following equations:

For $x=2$,

$$
\frac{1}{y}+\frac{1}{z}=-\frac{1}{7}
$$

For $x=3$,

$$
\frac{1}{y}+\frac{1}{z}=\frac{1}{42}
$$

For $x=4$,

$$
\frac{1}{y}+\frac{1}{z}=\frac{3}{28}
$$

For $x=5$,

$$
\frac{1}{y}+\frac{1}{z}=\frac{11}{70}
$$

For $x=6$,

$$
\frac{1}{y}+\frac{1}{z}=\frac{4}{21}
$$

For $x=8$,

$$
\frac{1}{y}+\frac{1}{z}=\frac{3}{14}
$$

We avoid Eq. (2.35) because it leads to negative solutions.

Solving Eqs. (2.36)-(2.40) by the above procedure, we get:

$$
\begin{aligned}
(x, y, z) \in & \{(3,43,1806),(3,44,924),(3,45,630),(3,48,483),(3,49,294),(6,6,42), \\
& (6,7,21),(6,9,18),(6,10,15),(6,12,12),(4,10,140),(4,12,42), \\
& (4,14,28),(5,7,70)\} .
\end{aligned}
$$

Finally, we solve Eq. (2.6), that is, Eq. (1.1) when $w=8$.

We observe that $\frac{1}{x}<\frac{3}{8} \Rightarrow x \geq 2$.

Under the assumption $x \leq y \leq z$, Eq. (2.6) gives $\frac{1}{x}+\frac{1}{y}+\frac{1}{z} \leq \frac{3}{x}$ and thus $x \leq 8$.

Hence $x \in\{2,3,4,5,6,7,8\}$ and thus Eq. (2.6) leads to the following equations:

For $x=2$,

$$
\frac{1}{x}+\frac{1}{y}=-\frac{1}{8}
$$


For $x=3$,

$$
\frac{1}{y}+\frac{1}{z}=\frac{1}{24}
$$

For $x=4$,

$$
\frac{1}{y}+\frac{1}{z}=\frac{1}{8}
$$

For $x=5$,

$$
\frac{1}{y}+\frac{1}{z}=\frac{7}{40}
$$

For $x=6$,

$$
\frac{1}{y}+\frac{1}{z}=\frac{5}{24},
$$

For $x=7$,

$$
\frac{1}{y}+\frac{1}{z}=\frac{13}{56}
$$

For $x=8$,

$$
\frac{1}{y}+\frac{1}{z}=\frac{1}{4}
$$

We avoid Eq. (2.41) because it leads to negative solutions.

Solving Eqs. (2.42)-(2.47) by the above procedure, we get:

$$
\begin{aligned}
(x, y, z) \in & \{(3,25,600),(3,26,312),(3,27,213),(3,28,168),(3,30,120),(3,32,96), \\
& (4,9,72),(4,10,40),(4,12,24),(4,16,16),(8,5,20),(8,6,12),(8,8,8), \\
& (5,6,120),(5,8,20),(6,9,72),(6,10,40),(6,12,24),(6,16,16)\} .
\end{aligned}
$$

The above solutions $(w, x, y, z)$ are found under the assumption $w \leq x \leq y \leq z$. Thus we can conclude that any permutation of $(w, x, y, z)$ is a solution of Eq. (1.1).

We now state the following theorem which follows the above discussion.

Theorem 2.1 The equation $\frac{1}{w}+\frac{1}{x}+\frac{1}{y}+\frac{1}{z}=\frac{1}{2}$ has only a finite number of solutions in the positive integers $w, x, y$, and $z$.

We now state and prove general results.

\section{Theorem 2.2 The Diophantine equation}

$$
\frac{1}{w}+\frac{1}{x}+\frac{1}{y}+\frac{1}{z}=\frac{m}{n}
$$


where $m, n>1$ are integers, has only a finite number of solutions in the positive integers $w, x, y$, and $z$.

Proof Let us assume that $w \leq x \leq y \leq z$. Then

$$
\begin{aligned}
\frac{4}{z} & \leq \frac{1}{w}+\frac{1}{x}+\frac{1}{y}+\frac{1}{z} \leq \frac{4}{w} \\
& \Rightarrow \frac{4}{z} \leq \frac{m}{n} \leq \frac{4}{w} \\
& \Rightarrow \frac{1}{z} \leq \frac{m}{4 n} \leq \frac{1}{w} \\
& \Rightarrow z \geq \frac{4 n}{m} \geq w .
\end{aligned}
$$

Again $\frac{1}{w}<\frac{m}{n}$ and thus $w>\frac{n}{m}$.

This shows that $w \in\left(\frac{n}{m}, \frac{4 n}{m}\right]$ and hence $x$ has only a finite number of integer values.

Now let $w=p_{1}$ be such an integer value. Then Eq. (2.48) gives

$$
\frac{1}{x}+\frac{1}{y}+\frac{1}{z}=\frac{m}{n}-\frac{1}{p_{1}}=\frac{p_{1} m-n}{p_{1} n}=\frac{m_{2}}{n_{2}} .
$$

Also, $x \leq y \leq z \Rightarrow \frac{3}{z} \leq \frac{m_{1}}{n_{1}} \leq \frac{3}{x} \Rightarrow x \leq \frac{3 n_{1}}{m_{1}} \leq z$.

But $x>\frac{n_{1}}{m_{1}}$ as $\frac{1}{x}<\frac{m_{1}}{n_{1}}$. Thus $x \in\left(\frac{n_{1}}{m_{1}}, \frac{3 n_{1}}{m_{1}}\right]$ and hence $x$ can take only a finite number of integer values. Let $x=p_{2}$ be such a value. Then Eq. (2.49) implies

$$
\frac{1}{y}+\frac{1}{z}=\frac{m_{1}}{n_{1}}-\frac{1}{p_{2}}=\frac{m_{2}}{n_{2}}
$$

Since $\frac{m_{2}}{n_{2}} \leq \frac{2}{y}$, so that $y \in\left[p_{2}, \frac{2 n_{2}}{m_{2}}\right]$ and thus $y$ can also take only a finite number of integer values. Finally, if $y=p_{3}$ is such a value, then Eq. (2.50) gives $z=\frac{p_{3} n_{2}}{p_{3} m-n_{2}}$. Thus the number of integer values of $z$ is also finite.

Following a similar procedure, we can also establish the following result.

Theorem 2.3 The Diophantine equation

$$
\frac{1}{x_{1}}+\frac{1}{x_{2}}+\frac{1}{x_{3}}+\cdots+\frac{1}{x_{n}}=\frac{p}{q}
$$

where $p, q>1$ are integers, has only a finite number of solutions in the positive integers $x_{1}, x_{2}, \ldots, x_{n}$.

\section{Conclusion}

In this paper, we explicitly find the solutions in positive integers $w, x, y$, and $z$ of the title equation. Applying an analogue procedure, we prove that the Diophantine equation

$$
\frac{1}{w}+\frac{1}{x}+\frac{1}{y}+\frac{1}{z}=\frac{m}{n}
$$

where $m, n>1$ are integers, has only a finite number of solutions in the positive integers $w, x, y$, and $z$. We finally claim that the same holds for Eq. (2.51). 


\section{Acknowledgements}

The author would like to thank all the members of her research group for their careful reading of this manuscript. The author would like to thank anonymous referees for carefully reading this manuscript and their valuable suggestions.

\section{Funding}

This work was supported by the Natural Science Basic Research Plan in Shaanxi Province of Chins (Grant no. 2016JM1012) and the Science Foundation of Baoji University of Arts and Sciences of China (Grant nos. ZK16069 and ZK11135).

\section{Competing interests}

The author declares that there are no competing interests.

\section{Authors' contributions}

The author provided the problems and gave the proof of the main results. The author also read and approved the final manuscript.

\section{Publisher's Note}

Springer Nature remains neutral with regard to jurisdictional claims in published maps and institutional affiliations.

Received: 8 May 2018 Accepted: 18 July 2018 Published online: 28 July 2018

\section{References}

1. Elsholtz, C.: Sums of $k$ unit fractions. Trans. Am. Math. Soc. 353(8), 3209-3227 (2001)

2. Elsholtz, C., Tao, T.: Counting the number of solutions to the Erdos-Straus equation on unit fractions. J. Aust. Math. Soc. 94(1), 50-105 (2013)

3. Erdos, P:: $A z \frac{1}{x_{1}}+\frac{1}{x_{2}}+\cdots+\frac{1}{x_{n}}$ egyenlet eges szamu megoldasairol. Mat. Lapok 1, 192-210 (1950)

4. Guy, R.K.: Unsolved Problems in Number Theory, 3rd edn. Springer, New York (2004)

5. Hagedorn, T.R.: A proof of a conjecture on Egyptian fractions. Am. Math. Mon. 107, 62-63 (2000)

6. Li, D.L.: Equation $\frac{4}{n}=\frac{1}{x}+\frac{1}{y}+\frac{1}{2}$. J. Number Theory 13(4), 485-494 (1981)

7. Mordell, L.J.: Diophantine Equations. Academic Press, London (1969)

8. Sander, J.W.: On $\frac{4}{n}=\frac{1}{x}+\frac{1}{y}+\frac{1}{2}$ and Iwaniec half dimensional sieve. J. Number Theory 46, 123-136 (1994)

9. Webb, W.A.: On $\frac{4}{n}=\frac{1}{x}+\frac{1}{y}+\frac{1}{z}$. Proc. Am. Math. Soc. 25(3), 578-584 (1970)

\section{Submit your manuscript to a SpringerOpen ${ }^{\circ}$ journal and benefit from:}

- Convenient online submission

- Rigorous peer review

- Open access: articles freely available online

- High visibility within the field

Retaining the copyright to your article 